Ferrata Storti Foundation

\section{Sphingolipid dysregulation due to lack of functional KDSR impairs proplatelet formation causing thrombocytopenia}

\author{
Tadbir K. Bariana, ${ }^{1.2 .3,4}$ Veerle Labarque, ${ }^{5}$ Jessica Heremans, ${ }^{5}$ Chantal Thys, ${ }^{4,5}$ \\ Mara De Reys, ${ }^{5}$ Daniel Greene, ${ }^{3,4,6,7}$ Benjamin Jenkins, ${ }^{8}$ Luigi Grassi, ${ }^{3,4,6,7}$ \\ Denis Seyres, ${ }^{3,4,6,7}$ Frances Burden, ${ }^{3,4,6}$ Deborah Whitehorn, ${ }^{3,4,6}$, \\ Olga Shamardina, ${ }^{3,4,6}$ Sofia Papadia, ${ }^{3,4,6}$ Keith Gomez, ${ }^{1,2,4}$ NIHR BioResource, ${ }^{4}$ \\ Chris Van Geet, ${ }^{4,5}$ Albert Koulman, ${ }^{8}$ Willem H. Ouwehand, ${ }^{3,4,6,9,10}$ \\ Cedric Ghevaert, ${ }^{3,6,9}$ Mattia Frontini, , ${ }^{3,4,6,9}$ Ernest Turro ${ }^{3,4,6,7}$ \\ and Kathleen Freson ${ }^{4,5}$
}

Volume 104(5):1036-1045

\section{Correspondence: \\ KATHLEEN FREESON \\ kathleen.freson@med.kuleuven.be \\ Received: August 17, 2018. \\ Accepted: November 19, 2018. \\ Pre-published: November 22, 2018.}

doi:10.3324/haematol.2018.204784

Check the online version for the most updated information on this article, online supplements, and information on authorship \& disclosures: www.haematologica.org/content/104/5/1036

(C)2019 Ferrata Storti Foundation

Material published in Haematologica is covered by copyright. All rights are reserved to the Ferrata Storti Foundation. Use of published material is allowed under the following terms and conditions:

https://creativecommons.org/licenses/by-nc/4.0/legalcode. Copies of published material are allowed for personal or internal use. Sharing published material for non-commercial purposes is subject to the following conditions:

https://creativecommons.org/licenses/by-nc/4.0/legalcode, sect. 3. Reproducing and sharing published material for commercial purposes is not allowed without permission in writing from the publisher.
${ }^{1}$ Department of Haematology, University College London, UK; ${ }^{2}$ The Katharine Dormandy Haemophilia Centre and Thrombosis Unit, Royal Free London NHS Foundation Trust, UK; ${ }^{3}$ Department of Haematology, University of Cambridge, Cambridge Biomedical Campus, UK; ${ }^{4}$ NIHR BioResource, Cambridge University Hospitals, Cambridge Biomedical Campus, UK; ${ }^{5}$ Department of Cardiovascular Sciences, Center for Molecular and Vascular Biology, University of Leuven, Belgium; ${ }^{6} \mathrm{NHS}$ Blood and Transplant, Cambridge Biomedical Campus, UK; ${ }^{7}$ Medical Research Council Biostatistics Unit, Cambridge Institute of Public Health, Cambridge Biomedical Campus, UK; ${ }^{8} \mathrm{NIHR}$ Biomedical Research Centre Core Metabolomics and Lipidomics Laboratory, University of Cambridge, Cambridge Biomedical Campus, UK; ${ }^{9}$ British Heart Foundation Centre of Excellence, Division of Cardiovascular Medicine, Cambridge University Hospitals, Cambridge Biomedical Campus, UK and ${ }^{10}$ Wellcome Sanger Institute, Wellcome Genome Campus, Hinxton, Cambridge, UK phingolipids are fundamental to membrane trafficking, apoptosis, and cell differentiation and proliferation. KDSR or 3-keto-dihydrosphingosine reductase is an essential enzyme for de novo sphingolipid synthesis, and pathogenic mutations in KDSR result in the severe skin disorder erythrokeratodermia variabilis et progressiva-4. Four of the eight reported cases also had thrombocytopenia but the underlying mechanism has remained unexplored. Here we expand upon the phenotypic spectrum of KDSR deficiency with studies in two siblings with novel compound heterozygous variants associated with thrombocytopenia, anemia, and minimal skin involvement. We report a novel phenotype of progressive juvenile myelofibrosis in the propositus, with spontaneous recovery of anemia and thrombocytopenia in the first decade of life. Examination of bone marrow biopsies showed megakaryocyte hyperproliferation and dysplasia. Megakaryocytes obtained by culture of CD34+ stem cells confirmed hyperproliferation and showed reduced proplatelet formation. The effect of KDSR insufficiency on the sphingolipid profile was unknown, and was explored in vivo and in vitro by a broad metabolomics screen that indicated activation of an in vivo compensatory pathway that leads to normalization of downstream metabolites such as ceramide. Differentiation of propositus-derived induced pluripotent stem cells to megakaryocytes followed by expression of functional KDSR showed correction of the aberrant cellular and biochemical phenotypes, corroborating the critical role of KDSR in proplatelet formation. Finally, Kdsr depletion in zebrafish recapitulated the thrombocytopenia and showed biochemical changes similar to those observed in the affected siblings. These studies support an important role for sphingolipids as regulators of cytoskeletal organization during megakaryopoiesis and proplatelet formation. 


\section{Introduction}

3-keto-dihydrosphingosine reductase (KDSR) is an early, essential enzyme in the pathway of de novo sphingolipid synthesis that catalyzes the conversion of 3-ketodihydrosphingosine (KDS) to dihydrosphingosine (DHS) on the cytosolic leaflet of the endoplasmic reticulum. ${ }^{1}$ The canonical transcript for KDSR encodes a 332 amino acid protein. The gene is widely transcribed, ${ }^{1-3}$ consistent with the integral roles of the sphingolipid family in forming lipid rafts that facilitate membrane trafficking and in the regulation of fundamental cellular functions that include apoptosis, differentiation, and proliferation. ${ }^{4}$ The importance of sphingolipid synthesis for normal cellular functions is illustrated by the complex multisystem phenotypes of null mice for key enzymes or receptors in the pathway, including defective platelet activation and thrombus formation. ${ }^{5,6}$ A pathway for de novo synthesis of sphingolipids in a megakaryocytic cell line has been shown, but this plays a minimal role in mature platelets, which instead acquire essential sphingolipids by incorporating them from plasma or recycling plasma membrane sphingomyelins, both largely independently of KDSR?

Consistent with these important roles of sphingolipids, compound heterozygous variants in KDSR (Figure 1) have recently been identified as causing the severe skin disorder erythrokeratodermia variabilis et progressiva 4 (EKVP4, OMIM617526), a condition characterized by neonatal onset of thick, scaly skin on the face and genitals, and milder erythematous palmo-plantar scaling. ${ }^{8}$ This observation established a role for KDSR in the homeostasis of keratinization; however, it was unclear whether these cases had hematologic pathologies. A more recent study described four probands with EKVP4 caused by KDSR variants accompanied by severe thrombocytopenia and platelet dysfunction in infancy. ${ }^{9}$ A reduction in plasma S1P and surface-exposed ceramide in human platelets, as well as diminished ceramide levels in affected skin, were reported. Bone marrow (BM) morphology in one patient was normal and in a second patient demonstrated increased megakaryopoiesis. For this patient, a diagnosis of immune-mediated thrombocytopenia was made with no response to corticosteroid treatment and minimal response to splenectomy. No further exploration of the molecular mechanism underlying the thrombocytopenia was undertaken. ${ }^{9}$

Here we provide evidence that in this pedigree, KDSR plays a fundamental role in megakaryopoiesis, cytoplasmic organization, and proplatelet formation. We describe a pedigree in which compound heterozygous variants in KDSR segregate with severe thrombocytopenia and minimal or no skin involvement. We report novel phenotypes of progressive juvenile myelofibrosis in the propositus, who is older, and anemia in both siblings. Broad metabolic profiling complemented by targeted mass spectrometry assays confirm KDSR hypofunction and suggest activation of an alternative, compensatory pathway in vivo. Depletion of kdsr in zebrafish and studies with $\mathrm{CD} 34^{+}$ stem cell- and induced pluripotent stem cell (iPSC)-derived MK show cellular and biochemical signatures in common with those observed in our patients, showing the mechanism by which $K D S R$ variants mediate thrombocytopenia.

\section{Methods}

\section{Recruitment and sequencing}

Following informed, written consent (ethical study approval ML3580), the propositus was recruited to the Bleeding, thrombotic and Platelet Disorders (BPD) domain of the NIHR BioResourceRare Diseases study (UK Research Ethics Committee 13/EE/0325, https://bioresource.nihr.ac.uk). Further details are provided in the Online Supplementary Methods.

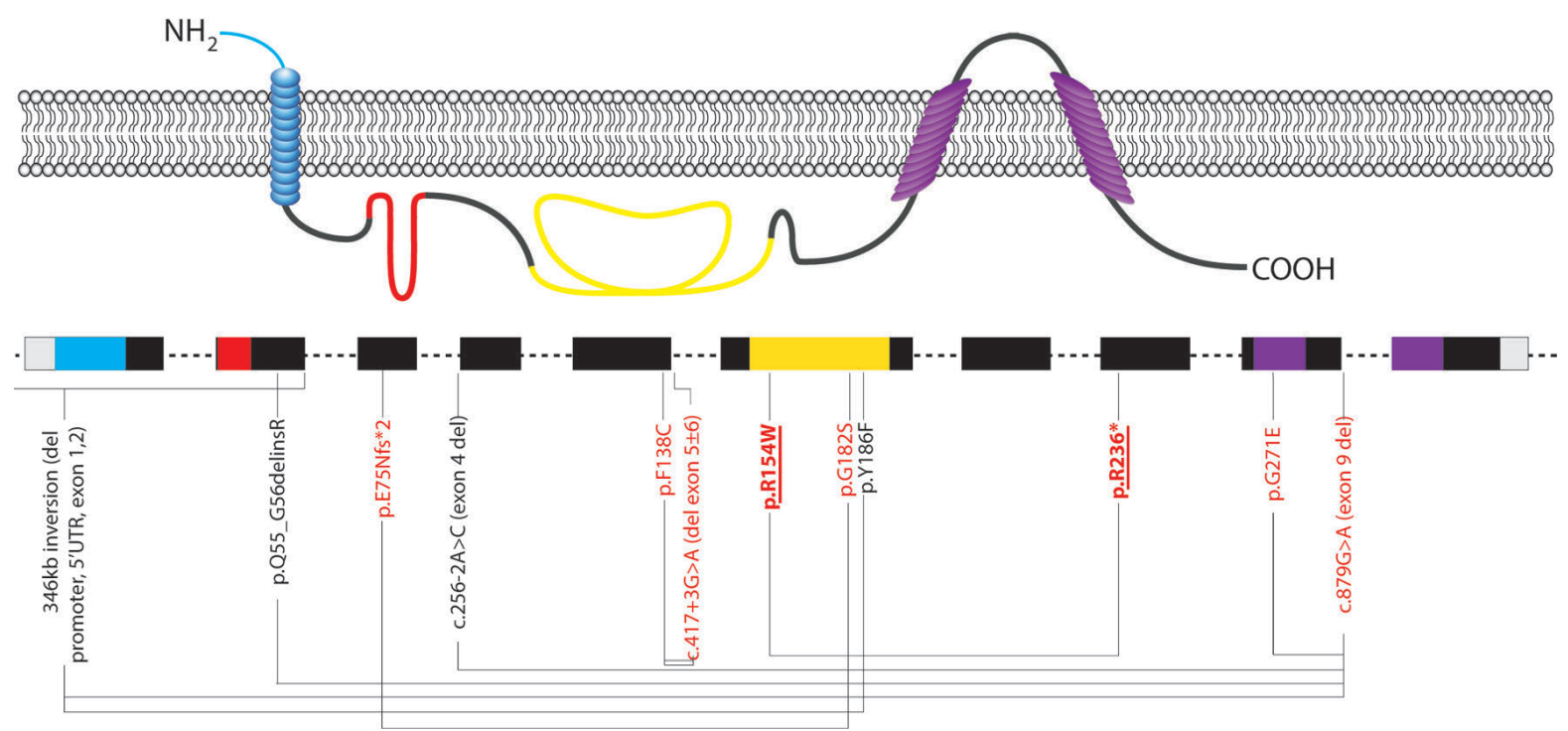

Figure 1. Reported KDSR variant genotypes and phenotypes in the context of 3-keto-dihydrosphingosine reductase (KDSR) structure and function. Protein and cDNA schematic adapted from Gupta et al. ${ }^{20}$ demonstrating location of known pathogenic KDSR variants with documented phenotypes in skin only (black), skin and platelets (red), and the novel variants reported in this manuscript in bold and underlined. Variants are linked by brackets where present in compound heterozygosity in an individual. Key structural elements of KDSR are illustrated: transmembrane anchors (blue, purple), the Rossman folds (red), and a highly conserved domain containing three putative catalytic sites (yellow). The novel p.Arg154Trp variant is within the catalytic domain. 


\section{Platelet studies}

Aggregation and transmission electron microscopy (EM) studies were performed as described previously. ${ }^{10}$

\section{Metabolic profiling}

Global metabolic profiling of plasma was performed by Metabolon Inc. (Durham, NC, USA) using the DiscoveryHD4 liq- uid chromatography tandem mass spectrometry (LC-MS/MS) platform, as previously described. ${ }^{11}$ Results of study participants were compared with 496 subjects between the ages of 4 and 55 years without thrombocytopenia. A separate LC-MS platform method, previously described, ${ }^{12,13}$ was used for specific confirmation of the global sphingolipid profile. Further details are provided in the Online Supplementary Methods.

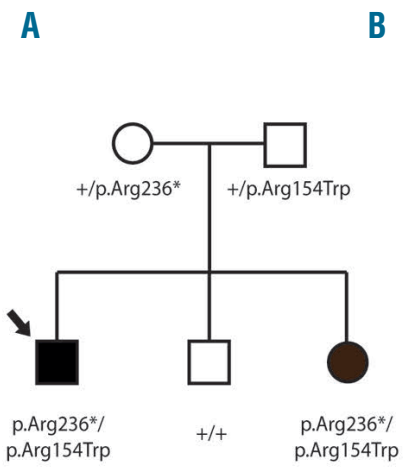

C

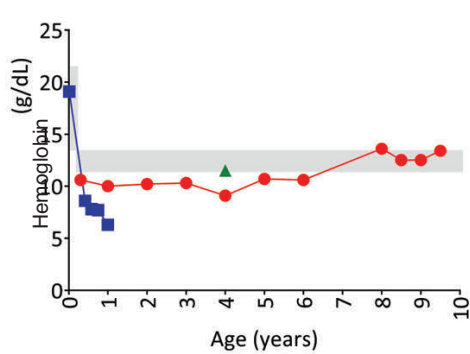

Age (years)

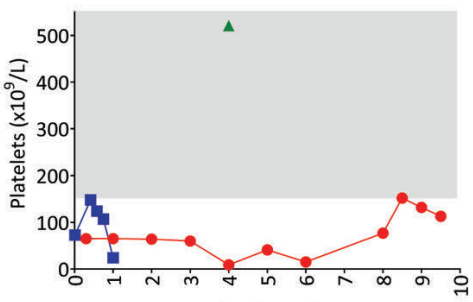

Age (years)

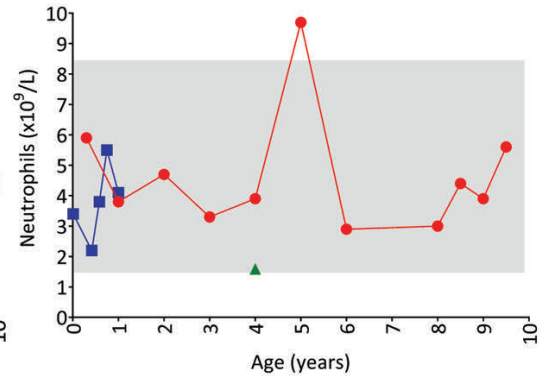

- Propositus

Sibling

- Healthy brother

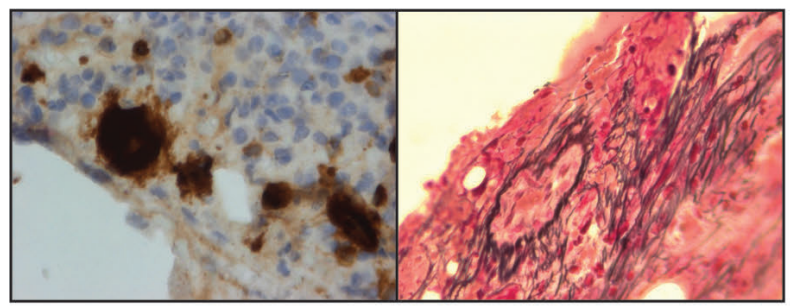

D

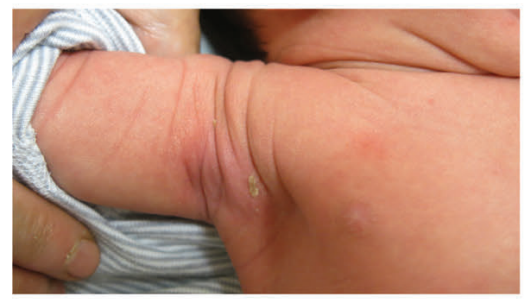

E
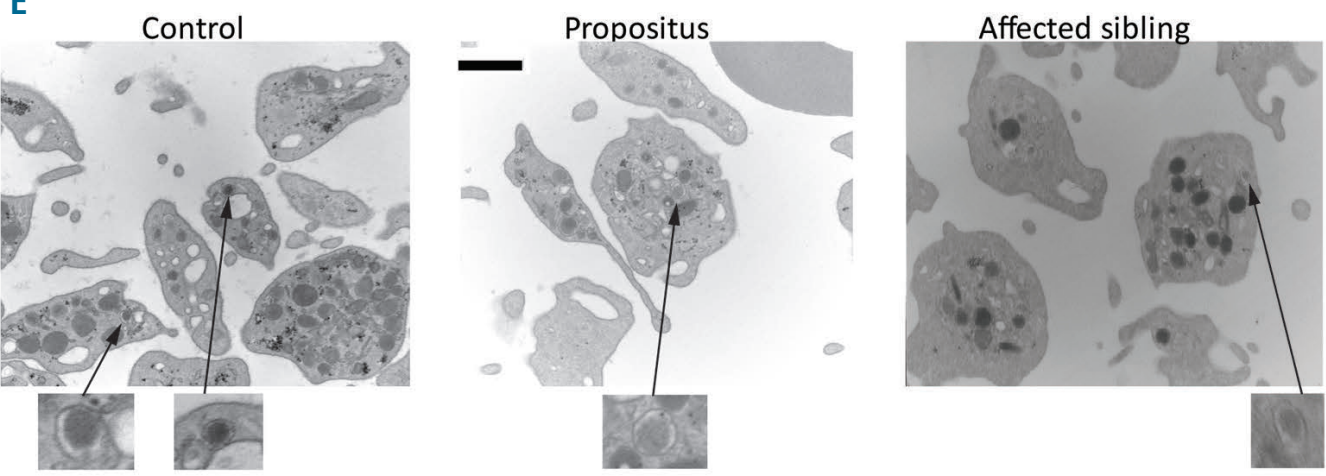

Figure 2. Clinical characteristics associated with loss-of-function KDSR variants. (A) Pedigree and variants identified in KDSR. ' + ' denotes the major allele. The propositus and affected sibling, but not the healthy sibling, carry the missense variant 18:61018270 G>A (p.Arg154Trp) and the nonsense variant 18:61006104 G>A (p.Arg236*). Co-segregation analysis demonstrated that the father carries the former and the mother the latter variant. (B) Serial blood counts are shown for the two affected siblings and a single value for the healthy brother. Fluctuating anemia and thrombocytopenia was observed, without evidence of neutropenia. (C) Bone marrow biopsy. (Left) Numerous dysplastic megakaryocytes made visible with linker for activation of T cell (LAT) staining are present. (Right) Marrow fibrosis with strong stromal reticulin staining. Magnification x40. Further images can be found in the Online Supplementary Appendix. (D) The affected sibling was born during the course of this study and presented at birth with thrombocytopenia and mild ichthyosis in her left axilla. The skin symptoms improved spontaneously over the first month. (E) Platelets were examined by electron microscopy for an unrelated healthy control, the propositus, and the affected sibling. There were no marked morphological differences. Arrowed magnifications show delta granules. Magnification x 12,000. 


\section{Stem cell differentiation assays}

CD34 ${ }^{+}$hematopoietic stem cells (HSC) were isolated by magnetic cell sorting (Miltenyi Biotec, Bergisch Gladback, Germany) from $\mathrm{BM}$ aspirates from the propositus (at 5 years of age) and an unrelated control, and from peripheral blood (PB) from the propositus (at 8.5 years of age), his affected sister (at 5 months of age), and an unrelated control.

In addition, expanded BM- and PM-derived ${ }^{12,13}$ HSC at day 3 of differentiation were used for liquid $\mathrm{MK}$ cultures in two experiments. In the first experiment, HSC obtained from the BM of the propositus were differentiated in parallel to a control. For the second experiment, HSC obtained from the PB of the propositus and his affected sister were cultured in parallel with a different control. Details of the differentiation protocols, colony assays and statistical analysis of megakaryocyte (MK) immunostaining are provided in the Online Supplementary Methods.

\section{Zebrafish analysis}

$\operatorname{Tg}(\mathrm{cd} 41: \mathrm{EGF})$ embryos ${ }^{14}$ were injected at the one-cell stage with a kdsr ATG morpholino (MO) (5' ctcagaggacatgggtcaacctgat, Kdsr$\mathrm{MO}$ ) purchased from Gene Tools LLC (Philomath, OR, USA) or with buffer (control). Zebrafish kdsr has ZFIN accession number
ZDB-GENE-040426-853. Thrombocyte formation was analyzed as described previously. ${ }^{15,16}$ Immunoblots were developed with goat anti-GFP (Rockland) and anti-FVT1/KDSR (Clone H-149; Santa Cruz, CA, USA). All animal protocols were approved by the Ethical Committee of KU Leuven, Belgium.

\section{Lentiviral reference 3-keto-dihydrosphingosine reductase transcript expression in induced pluripotent stem cells}

Induced pluripotent stem cells (iPSC) were prepared by the Cambridge Biomedical Research Centre iPSC core laboratory as described in the Online Supplementary Methods. iPSC were transduced with the lentiviral vector to express the open reading frame (ORF) of KDSR (pLenti-EF1a-KDSR-myc-DDK-IRES-Puro, Origene) and the un-cloned destination vector PS10085 (Origene) to generate the reference transcript rescue line (Kresc) and empty vector control line (Kev), respectively. The ORF is identical to transcript ENST00000591902 (RefSeq accession n. NM_002035, Origene TrueClone cDNA cat. RC201153), which has the highest reported expression in $\mathrm{MK}^{2}$. Details of lentiviral particle production, transduction and selection are given in the Online Supplementary Methods.

\section{A}
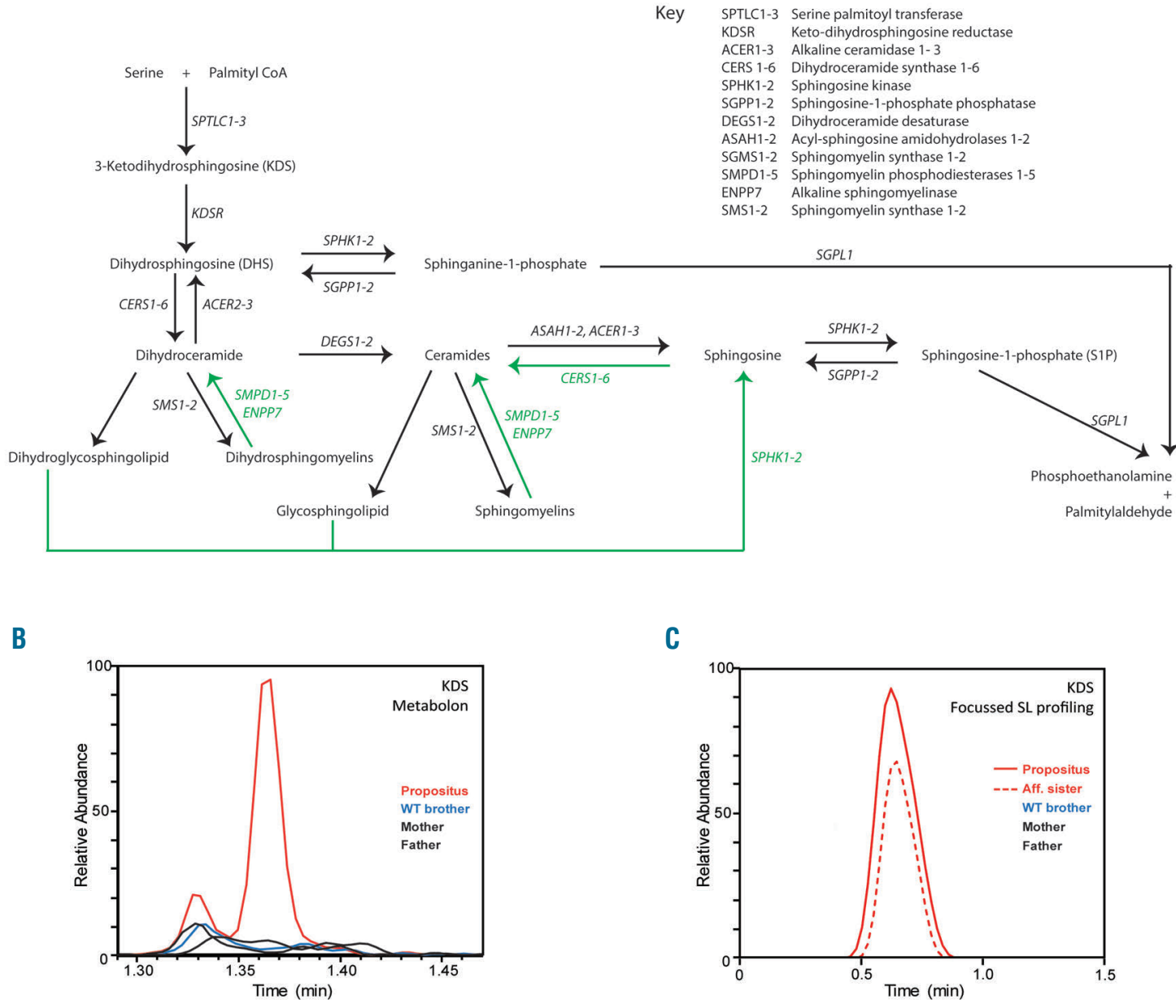

Palmitylaldehyde

Figure 3. Metabolic profiling shows that the KDSR variants are associated with loss-of-function and downstream sphingolipid pathway compensation. (A) Simplified sphingolipid pathway highlighting the role of the 3-keto-dihydrosphingosine reductase (KDSR) enzyme in de novo synthesis (black arrows) and the generation of sphingolipid intermediates from the recycling of complex sphingolipids and sphingomyelins (green arrows). (B) Mass spectrometry using the Metabolon platform shows the major chromatographic peak of 3-keto-dihydrosphingosine (KDS) in the plasma of the propositus, but not of the unaffected pedigree members (shown) or the controls (data not shown). (C) KDSR hypofunction was confirmed in the propositus and affected sister using a second mass spectrometry platform for targeted sphingolipid profiling. The chromatogram shows that KDS was detected in the plasma from the propositus and his affected sister but not in the plasma from the healthy brother, parents (shown), and two controls (data not shown). 


\section{Forward programming to MK}

Induced pluripotent stem cells were reprogrammed to $\mathrm{MK}$ (named iMK hereafter) using a protocol for generating MK described by Moreau et al. ${ }^{17} \mathrm{~K}^{\mathrm{resc}}$ and $\mathrm{K}^{\mathrm{ev}} \mathrm{iMK}$ were generated in three independent experiments. Details of iMK reprogramming, immunophenotyping, the proplatelet assay, and RNA sequencing are given in the Online Supplementary Methods.

\section{Results}

\section{Clinical characteristics}

The 8-year old male propositus was born to healthy, unrelated parents of European ancestry (Figure 2A). At 4 months of age he presented with a viral infection and was found to have a platelet count of $65 \times 10^{9} / \mathrm{L}$ and mild nor-
A

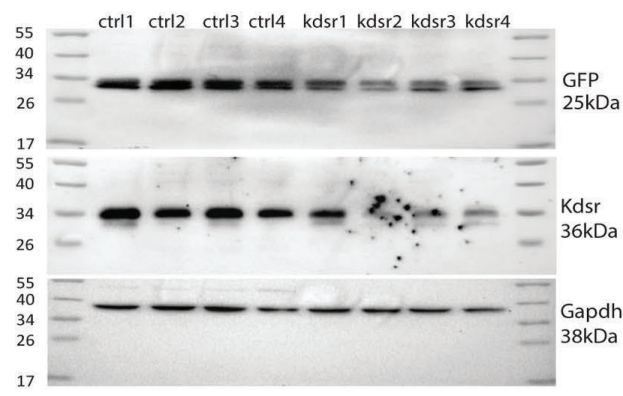

C

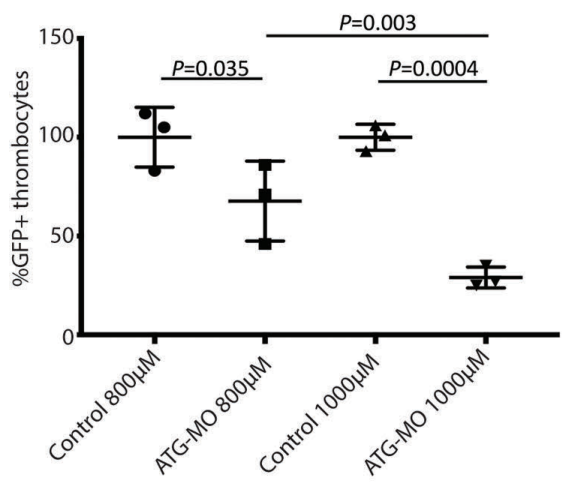

D

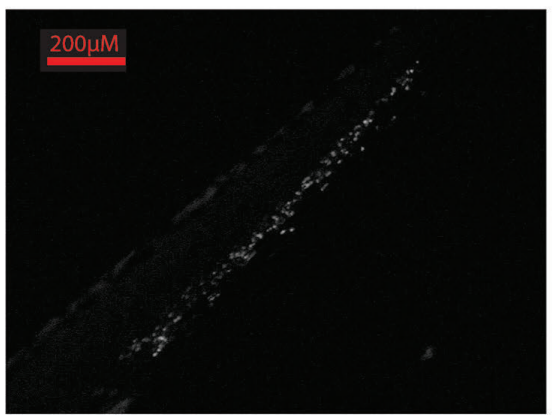

E

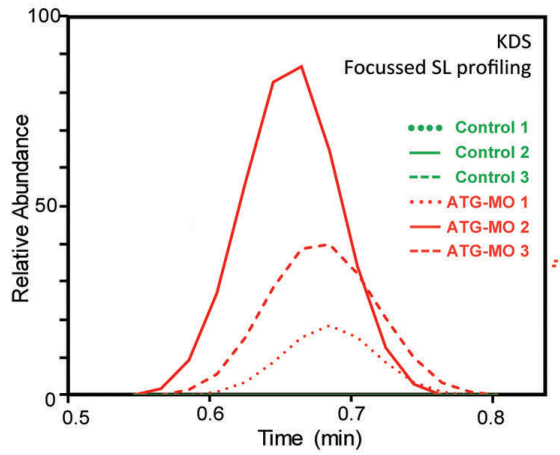

B

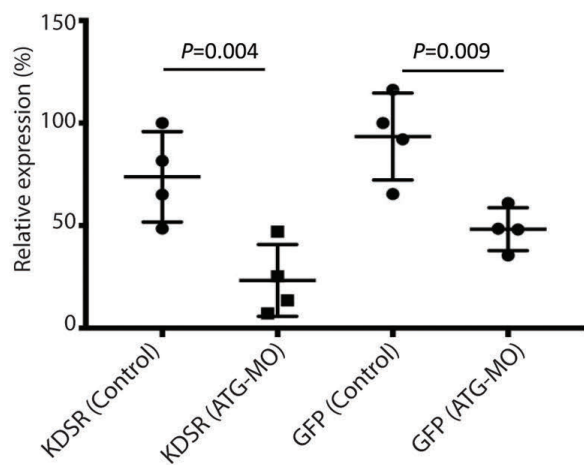

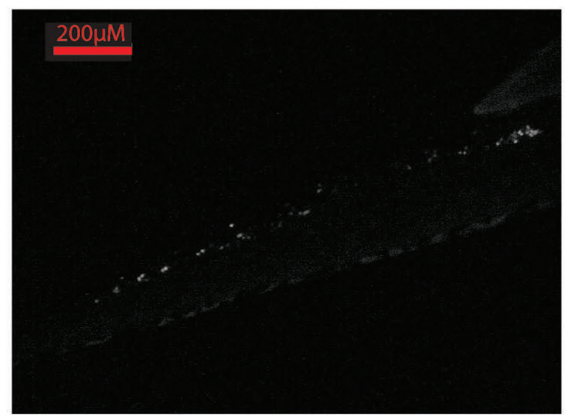

Figure 4. Kdsr morpholino knockdown is associated with reduced thrombopoiesis in zebrafish. (A) $\operatorname{Tg}(\mathrm{cd} 41$ :EGF) embryos were injected with a kdsr ATG-MO $(1000 \mu \mathrm{M})$ or with buffer (control). Embryos were lysed $72 \mathrm{hpf}$ and used for immunoblotting. GFP and Kdsr proteins were reduced in the kdsr knockdown condition. Equal amounts $(50 \mu \mathrm{g})$ were loaded ( 5 randomly selected embryos for each of the 4 conditions). Staining of Gapdh was used as loading control. (B) Quantification of immunoblot after normalization for Gapdh. Mean values are plotted and error bars show the standard deviation, analyzed by one-way ANOVA. (C) Quantification by flow cytometry of the number of GFP-labeled thrombocytes in Tg(cd41:EGFP) Danio rerio embryos at $72 \mathrm{hpf}$ for kdsr-MO (800 or $1000 \mu \mathrm{M})$ or buffer (control) injected fish. Values are means and Standard Deviations as quantified for 10 randomly selected embryos for each condition, performed in triplicate. Results were analyzed by one-way ANOVA. (D) Grayscale stereo-microscope images (x20 original magnification) in the tail region at $72 \mathrm{hpf}$ showed a reduced number of GFP-labeled thrombocytes (in white). (E) KDS levels in lysates from $72 \mathrm{hpf}$ embryos (20/condition) for kdsr$\mathrm{MO}(800 \mu \mathrm{M})$ or buffer (control) injected fish. KDS was detected in the lysates from the MO-injected embryos but not in the control-injected embryos. 
mocytic, normochromic anemia with normal iron and hematinic levels (Figure 2B, Online Supplementary Table S1A and Online Supplementary Figure S1). Subsequent complete blood counts showed on several occasions platelet counts $<100 \times 10^{9} / \mathrm{L}$ accompanied by rectal and gingival bleeding, excessive ecchymosis, and recurrent epistaxis when platelet counts were $<10 \times 10^{9} / \mathrm{L}$. Possible skin involvement was limited to a slow-to-heal perianal wound following rectal manometry. Serial BM examinations revealed increased numbers of dysplastic $M K$ and progressive severe myelofibrosis (Figure 2C, Online Supplementary Table $S 1 B$ and Online Supplementary Figure S2); despite this we observed significant fluctuation in the propositus's thrombocytopenia and normalization of the hemoglobin level over the course of his first decade (Figure 2B and Online Supplementary Table S1A). The mechanism of the improvement is unclear, and occurred in the absence of identifiable environmental, therapeutic, or dietary interventions. Genetic analyses of BM DNA excluded known somatic mutations causal of myelodysplasia or primary myelofibrosis (Online Supplementary Table S1B). Light transmission platelet aggregation was normal with the exception of an attenuated response to stimulation with collagen at low dose (Online Supplementary Table S1C). The propositus's older brother and his parents were unaffected (Figure 2A). His sister presented at birth with thrombocytopenia (Figure $2 \mathrm{~A}$ and $\mathrm{B}$, and Online Supplementary Table S1A) and mild ichthyosis in her left axilla (Figure 2D), but the skin symptoms resolved spontaneously over the first month. At 5 months of age she also developed persistent, normocytic, normochromic anemia (Figure 2B and Online Supplementary Table S1A). Transmission EM analysis showed platelets of normal size (Figure 2E). Delta (ठ)-granules appeared diminished; however, it was not possible to count these accurately in the absence of whole-mount EM or a specific $\delta$-granule marker (CD63 also stains lysosomal structures). There were no other marked ultrastructural abnormalities.

\section{Pathogenic variants in KDSR}

The propositus and his affected sister carry a maternally inherited nonsense variant 18:61006104 G>A (p.Arg236*) and a paternally inherited missense variant 18:61018270 G>A (p.Arg154Trp) in KDSR (Figure 2A). The variants were confirmed by Sanger sequencing ${ }^{18,19}$ (Online Supplementary Figure S3) and have minor allele frequencies in Europeans of $4.82 \times 10^{-5}$ and $2.32 \times 10^{-4}$, respectively. The missense variant p.Arg154Trp is localized in the catalytic domain of KDSR (Figure 1$)^{20}$ and both are found in the most abundant KDSR transcripts in MK (Online Supplementary Figure S4). ${ }^{2}$ The nonsense variant is absent from two out of three major platelet transcripts, which is in keeping with minimal de novo sphingolipid synthesis in mature platelets (Online Supplementary Figure S4). ${ }^{7}$ The results of co-segregation study were concordant with an autosomal recessive mode of inheritance (Figure 2A).

\section{Sphingolipid profiles}

We reasoned that the variants would cause reduced enzymatic function, leading to a build-up of the substrate KDS (Figure 3A). Indeed, global metabolic profiling showed KDS to be detectable in plasma from the proposi-
A

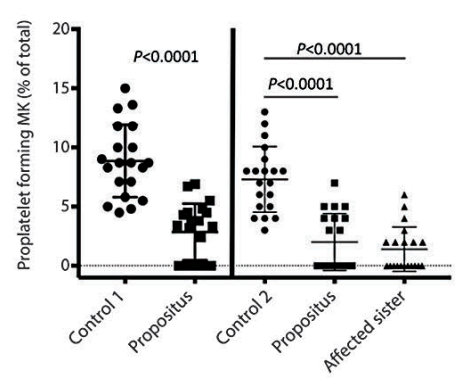

B
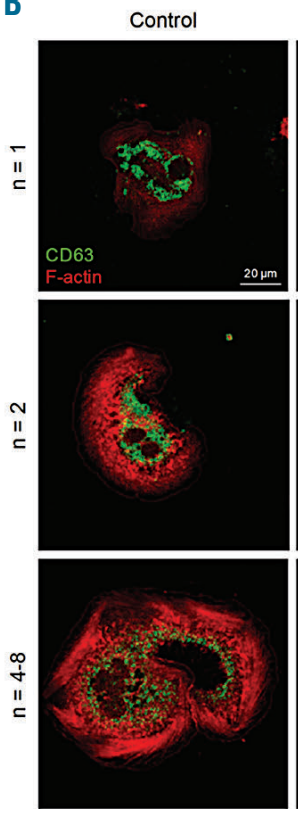

Propositus

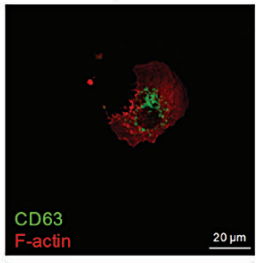

C

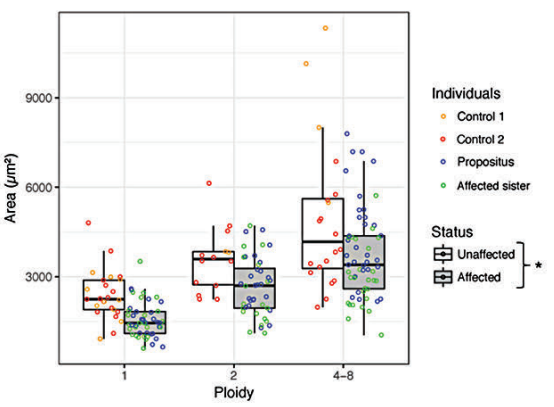

Figure 5. KDSR variants are associated with reduced proplatelet formation by megakaryocytes (MK). (A) Quantification of proplatelet formation by MK at day 11 of differentiation. On the left are the results of differentiation of bone marrow (BM)-derived hematopoietic stem cells (HSC) from the propositus and control. (Right) Results of differentiation of HSC obtained from the blood of the propositus, his affected sibling, and a second control. All MK with proplatelets and membrane budding were counted as positive. Values plotted are means and Standard Deviations (SD) as quantified on 20 images. Results were analyzed by the unpaired, twotailed $t$-test. (B) MK at day 11 derived from BM HSC from the propositus and a control. MK are stained for the cytoskeletal marker F-actin (red) and lysosome and delta granule marker CD63 (green). MK from the propositus and affected sibling have irregular cytoskeletal structures with lamellipodia (arrows). Further images can be found in Online Supplementary Figure S10. (C) MK area was quantified by automated analysis. Modeling was performed using a linear mixed effects model and associated $P$-values were computed by a likelihood ratio test. MK from affected cases were smaller compared to unrelated controls $(P=0.01473)$. 
A

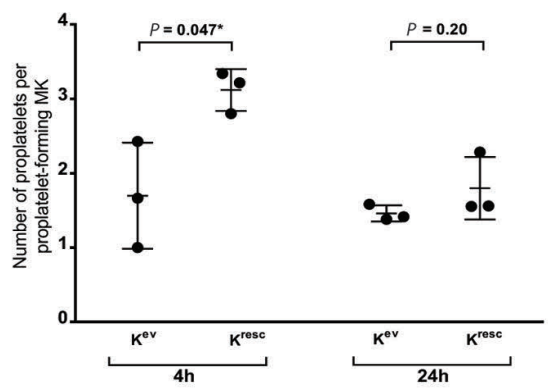

C
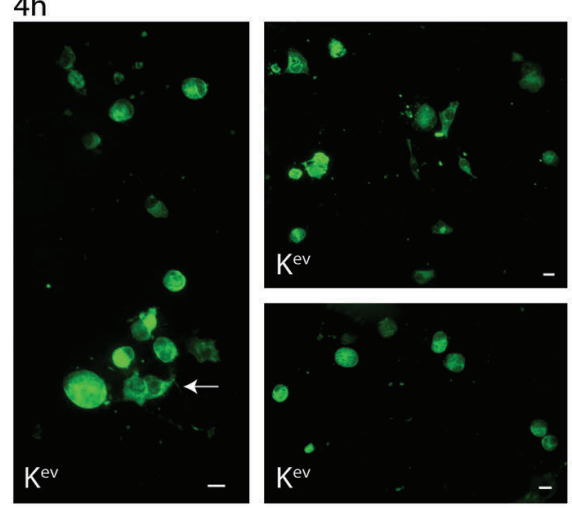

$24 \mathrm{~h}$
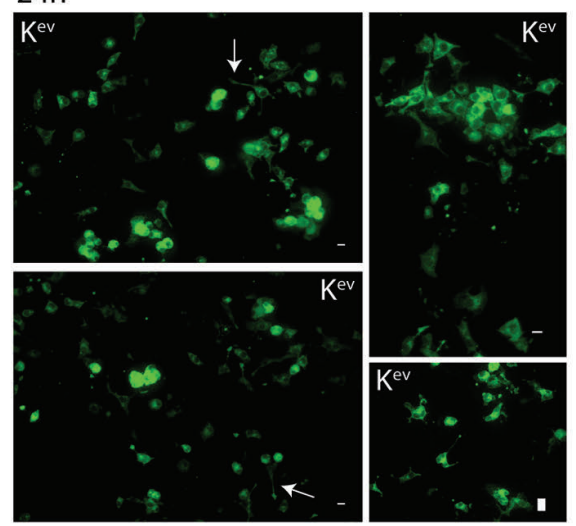

a-tubulin +/- DAPI

D
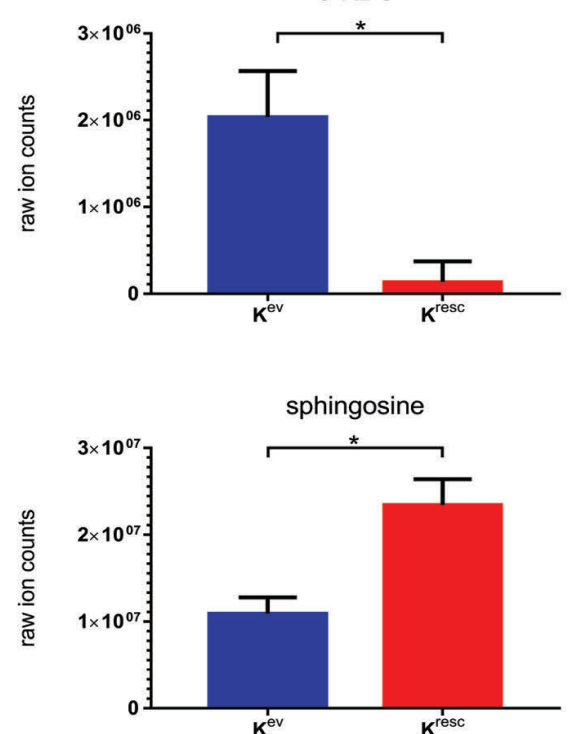

B
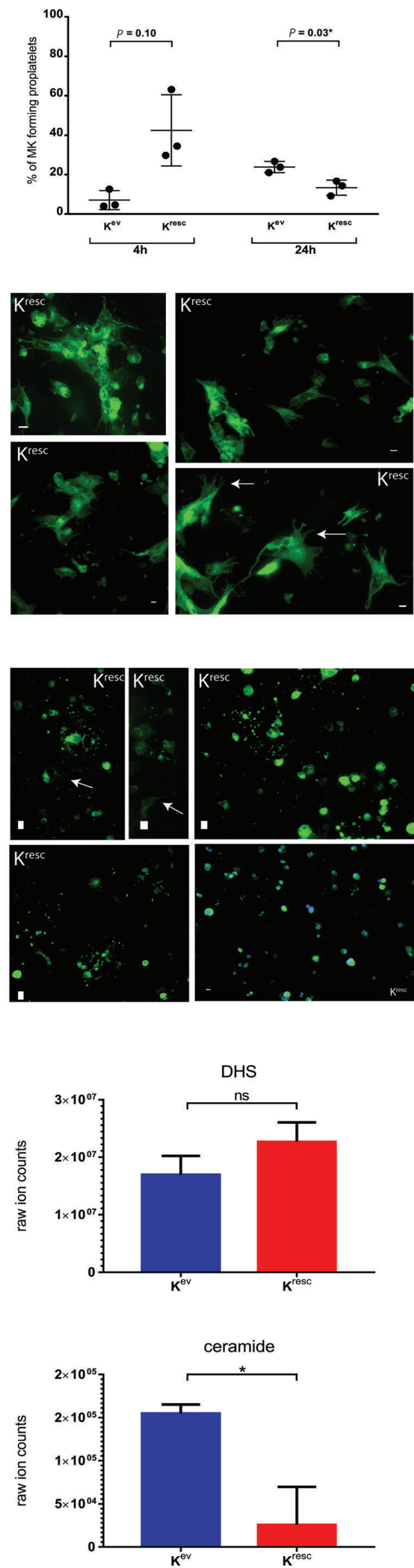

Figure 6. KDSR reference allele expression rescues ineffective proplatelet formation. Proplatelet formation by induced pluripotent stem cells reprogrammed to MK (iMK). One hundred percent of live MK plated for the proplatelet assay were CD41 positive and $75 \%$ were dual positive for CD41 and CD42 by flow cytometry (Online Supplementary Figure S11A). Cytoskeletal marker $\alpha$-tubulin was stained with antibodies in green and nuclei were stained with DAPI in blue. Proplatelet formation was counted manually. Values shown were analyzed using the paired, two-tailed Student $t$-test plotted as means and standard deviations. $* P<0.05$ was considered statistically significant. (A) The number of proplatelets formed at 4 hours (h) per proplatelet-forming $\mathrm{MK}$ (PPFMK) by the rescued and non-rescued iMK. The differences were significant at $4 \mathrm{~h}$ $(P=0.047)$ but not at $24 \mathrm{~h}$ $(P=0.20)$. (B) There was no significant difference in the number of PPFMK- at $4 \mathrm{~h}$ between the two groups $(7 \cdot 1 \%$ vs. $42.4 \% ; P=0.10$ ), but at $24 \mathrm{~h}$ the rescued iMK showed less PPFMK $\quad(P=0.03)$. (C) Representative images from the proplatelet formation assay at 4 and $24 \mathrm{~h}$. Proplatelets are indicated by white arrows. (Top left and right) Results at $4 \mathrm{~h}$ for rescued iMK show increased proplatelet formation. White scale bars indicate $10 \mu \mathrm{m}$. (Bottom left and right) Results at $24 \mathrm{~h}$ show little proplatelet formation for the rescued iMK, and residual cells are either fragmented into platelet-like particles, or consist of bare nuclei. (D) Metabolon mass spectrometry results for non-rescued and rescued iMK. The ion counts for KDS detection differed significantly between the non-rescued and non-rescued iMK $(P=0.02)$. There was no difference in DHS level between the groups of samples but the levels of sphingosine and ceramide were lowe and higher in the non-rescued versus rescued iMK, respectively. ns: not significant. 
tus but not from the parents, the healthy sibling, or 496 unrelated controls (Figure $3 \mathrm{~B}$ ). This finding was corroborated using a LC-MS platform for the selective measurement of specific sphingolipids, which confirmed that KDS was detectable in both patients and absent from the plasma of controls (Figure 3C and Online Supplementary Figure S5). Interestingly, there was no reduction in downstream sphingolipids in the patients using either platform (Online Supplementary Tables S2 and S3A). In fact, global profiling showed levels of the KDSR product, DHS, were higher for the propositus than controls. These findings raise the hypothesis that KDSR hypofunction during de novo sphingolipid synthesis is compensated in vivo by alternative mechanisms, for example, by the recycling of relatively abundant sphingomyelins along a pathway that normally contributes little to free DHS production. ${ }^{4}$

\section{Depletion of $\mathbf{k d s r}$ in zebrafish causes thrombocytopenia}

We explored the role of the enzyme on thrombocyte formation in zebrafish by $\mathrm{MO}$-mediated depletion of the $k d s r$ transcript in $\mathrm{Tg}(\mathrm{cd} 41: \mathrm{EGFP})$. As expected, this led to a reduction of the Kdsr protein level (Figure $4 \mathrm{~A}$ and $\mathrm{B}$ ) and resulted in curved tails, which is a typical feature for embryos with thrombocytopenia (Online Supplementary Figure S6). ${ }^{15}$ The number of thrombocytes was inversely correlated with the dose of $\mathrm{MO}$ injected (Figure 4C and D). Targeted sphingolipid profiling showed elevated and undetectable KDS in lysates from $\mathrm{MO}$ and control embryos, respectively (Figure 4E). Similar to the results obtained with the propositus's plasma, dihydroceramides, ceramides, sphingomyelins and glycosphingolipids that are downstream of Kdsr were not significantly different between Kdsr-depleted and control fish (Online Supplementary Table S3B).

\section{Impaired proplatelet formation in patient megakaryocytes}

CFU-GEMM cultures differentiated from bone marrow HSC of the propositus showed hyperproliferation of myeloid cells ( $P=0.001$, $t$-test $)$ with a reduced myeloid/erythroid ratio compared to the controls (Online Supplementary Figure S7). CFU-MK numbers were comparable to those of the control, although individual $\mathrm{MK}$ colonies were denser for the propositus, and liquid cultures showed an increased number of $\mathrm{MK}$ (Online Supplementary Figure S8). MK in control cultures formed proplatelets, whilst $\mathrm{MK}$ derived from both the propositus and the affected sister showed a strong reduction in proplatelet formation, despite similar levels of membrane budding and a higher number of CD41 and CD42 positive cells in propositus-derived cultures when compared with control MK (Figure 5A and Online Supplementary Figures S8-S10). Patient-derived MK also showed extensive, abnormal formation of lamellipodia and reduced cell size $(P=0.014$, likelihood ratio test) (Figure $5 \mathrm{~B}$ and $\mathrm{C})$.

\section{The abnormal morphological, functional, and biochemical features of the propositus's induced pluripotent stem cells reprogrammed to megakaryocytes can be rescued}

To corroborate the atypical phenotypes of MK derived from the HSC, we transduced iPSC from the propositus with lentiviral vectors containing the reference KDSR ORF $\left(\mathrm{K}^{\mathrm{resc}}\right)$ or an inert control vector $\left(\mathrm{K}^{\mathrm{ev}}\right)$, and reprogrammed these cells to iMK (Online Supplementary Figure S11A). Analysis of the iMK RNA-seq results showed similar KDSR gene expression but the majority of sequencing reads in the rescued $\mathrm{iMK}$ carried the reference allele at Chr18:61018270 G>A (p.Arg154Trp) (Online Supplementary Figure $S 11 B-D)$. These findings are consistent with correction of the genetic defect without significant overexpression, and resulted in increased proplatelet formation compared with control iMK $4 \mathrm{~h}$ after seeding $(P=0.047$, $t$-test) (Figure 6A-C). The observed iMK proplatelets were shorter and less branched than those observed following directed differentiation from stem cell cells, in keeping with published reports using this protocol. ${ }^{17}$ Upon microscopic inspection, the rescued iMK seemed larger than the non-rescued ones, which was confirmed to be significant by flow cytometry (Online Supplementary Figure S11E) and the increased proplatelet formation resulted by $24 \mathrm{~h}$ in little residual cytoplasm for the rescued versus the non-rescued iMK (Figure 6C). At the biochemical level, the rescue resulted in a significant reduction in KDS levels ( $P=0.02, t$-test) showing the effectiveness of the gene therapy approach in 'curing' the iMK from the propositus (Figure 6D). Similar to findings in plasma and in zebrafish, there was no difference in DHS levels between the iMK with and without functional KDSR transcripts, indicating that the postulated, compensatory mechanism is also present in iMK. We searched the iMK transcriptome landscape for possible differences in the levels of transcripts of other key enzymes that regulate sphingolipid synthesis and recycling (the enzymes examined are as shown in Figure $3 \mathrm{~A}$ ). This identified only ASAH1 and CERS6 transcripts to be down- and up-regulated respectively (posterior probabilities 0.610 and 0.774 ; log-fold change -0.67 and +0.70 , respectively). These two enzymes regulate the ceramide-sphingosine ratio (Figure 3A and Online Supplementary Figure S12), and in keeping with these findings, the rescued iMK showed higher sphingosine and lower ceramide levels (Figure 6D).

\section{Discussion}

Pathogenic mutations in KDSR have recently been associated with a recessively inherited syndrome of moderateto-severe skin pathology and thrombocytopenia. We have described two novel KDSR mutations causing thrombocytopenia in the propositus and his infant sister, expanding the phenotypic spectrum of this recently identified Mendelian disorder from severe skin pathology with no apparent hematologic involvement to profound thrombocytopenia and moderate anemia with spontaneous improvement across the first decade, and almost imperceptible dermatological abnormalities. In the propositus, BM studies also showed the novel phenotype of severe juvenile myelofibrosis; however, the sister was too young to allow confirmation of this phenotype. The biochemical sphingolipid signatures of the plasma and patient-derived $\mathrm{iMK}$ confirmed the predicted reduction in function with elevated levels of its substrate, KDS. This is, as expected, from the combination of a variant encoding a premature stop codon and a hypomorphic allele involving a missense variant in the catalytic domain. Unexpectedly, downstream metabolites in the sphingolipid pathway, including DHS, ceramide, and sphingosine-1-phosphate, were not reduced in plasma, suggesting that KDSR hypofunction 
during de novo sphingolipid synthesis is compensated by an alternative pathway. One possible alternative pathway is the recycling of relatively abundant sphingomyelins; a pathway previously shown to contribute to production of downstream sphingolipids such as dihydroceramide, ceramide, and sphingosine, but considered to contribute little, if at all, to DHS production under normal conditions. ${ }^{21}$ Post-translational modifications to sphingolipid enzymes may also explain the metabolic profile, and further work is required to explore this possibility. Importantly, the profile of increased ceramide and reduced sphingosine in propositus-derived iMK compared with rescued iMK, and the consistent and potentially explanatory transcriptional dysregulation of enzymes $A S A H 1$ and CERS6, is in contrast to the limited sphingolipid quantitation undertaken in previous studies which showed that ceramide levels were reduced in affected skin and that platelet surface exposure of ceramide was impaired in individuals with hypo-functional KDSR variants. ${ }^{9}$

Our observation that MK lacking functional KDSR are hyperproliferative is consistent with earlier reports, ${ }^{9}$ but we expand on this characterization by showing the $e x$ vivo generated patient $\mathrm{MK}$ to be smaller than controls and to be less effective in proplatelet formation. Proplatelets are pseudopodial projections of megakaryocyte cytoplasm, supported at their core by microtubular bundles that carry granules and other platelet cargo from the body of the megakaryocyte to the tip of the proplatelet. ${ }^{22}$ Aberrant size and proplatelet formation were not only observed in MK obtained by differentiation of primary HSC obtained from the two patients, but are also present in iMK generated by forward programming of iPSC derived from the propositus's fibroblasts. Taken together, we consider the ineffective platelet formation caused by the absence of KDSR to be the primary cause of the thrombocytopenia. Increased turnover because of a reduced platelet lifespan seems to be a less likely explanation because the immature platelet fraction was not significantly raised in the two patients compared with healthy controls (data not shown). We hypothesize that impaired platelet formation may, in turn, be caused by cytoskeletal disorganization and further experiments are required to explore this possibility. Pathogenic mutations in several other genes (e.g. MYH9, ACTN1, FLNA, TUBB1, DIAPH1, TPM4) encoding proteins with important functional roles in cytoskeletal reorganization and actin polymerization cause dominant forms of thrombocytopenia. ${ }^{23}$ However, these genetic disorders are characterized by enlarged platelets, and the mean volumes of the platelets of our patients are within the normal ranges for males and females, respectively.

The increased level of KDS in plasma was confirmed at the cellular level in iMK derived from the propositus. This increased level was normalized upon rescue of the propositus's iMK with a KDSR transcript carrying the reference allele. The correction of the biochemical defect was mirrored by a recovery of iMK size and improvement of their capacity to form proplatelets. To further support the central importance of KDSR in thrombopoiesis, we show that KDSR knockdown in a zebrafish model is associated with impaired thrombocyte formation. Similar approaches have identified multiple potential regulators of thrombopoiesis, ${ }^{24}$ though in isolation zebrafish studies these are limited by inherent differences in thrombopoiesis between mammals and fish, notably that zebrafish have nucleate thrombocytes rather than MK.

The marked, spontaneous improvement in the propositus's thrombocytopenia and anemia led to a reversal of the decision to treat the condition by HSC transplantation. The mechanism of this improvement is unclear, given the presence of progressive myelofibrosis and in the absence of clinical features to suggest significant extramedullary hemopoiesis such as splenomegaly. Recent studies have shown spontaneous improvement in blood counts in other inherited juvenile BM failure syndromes, most notably those associated with pathogenic variants in $S A M D 9$ or $S A M D 9 L{ }^{25}$ In these cases, the improvement was attributed to the acquisition of corrective somatic mutations. Further longitudinal studies of individuals affected by pathogenic KDSR variants is essential to determine whether the clinical course described is representative, and whether a careful watch-and-wait approach rather than early intervention may be more appropriate in this genetically-defined subgroup of cases with inherited thrombocytopenia accompanied by BM failure.

\section{Funding}

TKB is supported by the British Society of Haematology and NHS Blood and Transplant. KF and CVG are supported by the Fund for Scientific Research-Flanders (FWO Vlaanderen, Belgium; G.OB17.13N) and by the Research Council of the University of Leuven (BOF KU Leuven, Belgium; OT/14/098). C.V.G is holder of the Bayer and Norbert Heimburger (CSL Behring) Chairs. The structured illumination microscope was acquired through a CLME grant from Minister Lieten to the VIB BioImaging Core, Leuven. J.H is supported by the Fund for Scientific Research-Flanders (FWO Vlaanderen, Belgium) grant no.1S00816N. AK and BJ are funded by the National Institute for Health Research (NIHR) Biomedical Research Centre (RG64245). MF is supported by the British Heart Foundation (BHF) Cambridge Centre of Excellence (RE/13/6/30180). The Ouwehand laboratory receives support from the BHF, BristolMyers Squibb, European Commission, MRC, NHS Blood and Transplant, Rosetrees Trust, the NIHR Biomedical Research Centre based at Cambridge University Hospitals NHS Foundation Trust, and the University of Cambridge.

\section{Acknowledgments}

The NIHR BioResource - Rare Disease Study is a multicenter whole-genome sequencing (WGS) study of approximately 13,000 patients. The genotype and phenotype data are being incorporated in the 100,000 Genomes Project. This study makes use of data generated by the NIHR BioResource and a full list of investigators who contributed to the generation of the data is available from https://bioresource.nihr.ac.uk/rare-diseases/consortia-lists/. Funding for the project was provided by the NIHR (grant number RG65966). The NIHR BioResource projects were approved by Research Ethics Committees in the UK and appropriate national ethics authorities in non-UK enrollment centers. The authors are also grateful to all the research participants who donated their samples for this study and to Professor Andrew Mumford (University of Bristol, UK), Professor Michael Laffan (Imperial College London, UK), Dr Lining Guo (Metabolon Inc., Durham, NC, USA), and Dr Sergio Rodriguez-Cuenca and Professor Antonio Vidal-Puig from the University of Cambridge (UK) for their input. The $\mathrm{Tg}(\mathrm{cd} 41: E G F) 11$ line was a gift from Professor Leonard Zon (Hematology Division, Brigham and Women's Hospital, Boston, MA, USA). 


\section{References}

1. Kihara A, Igarashi Y. FVT-1 is a mammalian 3-ketodihydrosphingosine reductase with an active site that faces the cytosolic side of the endoplasmic reticulum membrane. J Biol Chem. 2004; 279(47):49243-49250.

2. Chen L, Kostadima M, Martens JH, et al. Transcriptional diversity during lineage commitment of human blood progenitors. Science. 2014;345(6204):1251033.

3. Battle A, Brown CD, Engelhardt BE, Montgomery SB. Genetic effects on gene expression across human tissues. Nature. 2017;550(7675):204-213.

4. Hannun YA, Luberto C, Argraves KM. Enzymes of sphingolipid metabolism: from modular to integrative signaling. Biochemistry. 2001;40(16):4893-4903.

5. Munzer P, Schmid E, Walker B, et al. Sphingosine kinase 1 (Sphk1) negatively regulates platelet activation and thrombus formation. Am J Physiol Cell Physiol. 2014;307(10):C920-927.

6. Munzer P, Borst $O$, Walker B, et al. Acid sphingomyelinase regulates platelet cell membrane scrambling, secretion, and thrombus formation. Arterioscler Thromb Vasc Biol. 2014;34(1):61-71.

7. Tani M, Sano $T$, Ito $M$, Igarashi $Y$. Mechanisms of sphingosine and sphingosine 1-phosphate generation in human platelets. J Lipid Res. 2005; 46(11):24582467.

8. Boyden LM, Vincent NG, Zhou J, et al. Mutations in KDSR cause recessive progressive symmetric erythrokeratoderma. Am J Hum Genet. 2017;100(6):978-984.

9. Takeichi T, Torrelo A, Lee JYW, et al. Biallelic mutations in KDSR disrupt ceramide synthesis and result in a spectrum of keratinization disorders associated with thrombocytopenia. J Invest Dermatol. 2017;137(11):2344-2353

10. Freson K, De Vos R, Wittevrongel C, et al. The TUBB1 Q43P functional polymorphism reduces the risk of cardiovascular disease in men by modulating platelet function and structure. Blood. 2005; 106(7):2356-2362.

11. Long $\mathrm{T}$, Hicks $\mathrm{M}$, Yu HC, et al. Wholegenome sequencing identifies common-torare variants associated with human blood metabolites. Nat Genet. 2017;49(4):568 578.

12. Koulman A, Woffendin G, Narayana VK, Welchman H, Crone C, Volmer DA. Highresolution extracted ion chromatography, a new tool for metabolomics and lipidomics using a second-generation orbitrap mass spectrometer. Rapid Commun Mass Spectrom. 2009; 23(10):1411-1418.

13. Lu L, Koulman A, Petry CJ, et al. An unbiased lipidomics approach Identifies Early second trimester lipids predictive of maternal glycemic traits and gestational diabetes mellitus. Diabetes Care. 2016;39(12):22322239

14. Lin HF, Traver D, Zhu H, et al. Analysis of thrombocyte development in CD41-GFP transgenic zebrafish. Blood. 2005; 106(12):3803-3810.

15. Louwette S, Labarque V, Wittevrongel C, et al. Regulator of G-protein signaling 18 controls megakaryopoiesis and the cilia-mediated vertebrate mechanosensory system. FASEB J. 2012;26(5):2125-2136.

16. Louwette S, Regal L, Wittevrongel C, et al. NPC1 defect results in abnormal platelet formation and function: studies in Niemann-Pick disease type $\mathrm{C} 1$ patients and zebrafish. Hum Mol Genet.2013;22(1):6173.

17. Moreau T, Evans AL, Vasquez L, et al
Large-scale production of megakaryocytes from human pluripotent stem cells by chemically defined forward programming Nat Commun. 2016;7(11208.

18. Lek M, Karczewski KJ, Minikel EV, et al Analysis of protein-coding genetic variation in 60,706 humans. Nature. 2016; 536(7616):285-291

19. Kircher M, Witten DM, Jain P, O'Roak BJ Cooper GM, Shendure J. A general framework for estimating the relative pathogenicity of human genetic variants. Nat Genet. 2014:46(3):310-315.

20. Gupta SD, Gable K, Han G, et al. Tsc10p and FVT1: topologically distinct shortchain reductases required for long-chain base synthesis in yeast and mammals. J Lipid Res. 2009;50(8):1630-1640.

21. Kitatani K, Idkowiak-Baldys J, Hannun YA The sphingolipid salvage pathway in ceramide metabolism and signaling. Cell Signal. 2008;20(6):1010-1018.

22. Italiano JE Jr, Lecine $P$, Shivdasani RA Hartwig JH. Blood platelets are assembled principally at the ends of proplatelet processes produced by differentiated megakaryocytes. J Cell Biol. 1999 147(6):1299-1312.

23. Lentaigne C, Freson K, Laffan MA, Turro E Ouwehand WH. Inherited platelet disorders: toward DNA-based diagnosis. Blood. 2016;127(23):2814-2823.

24. Bielczyk-Maczynska E, Serbanovic-Canic J Ferreira L, et al. A loss of function screen of identified genome-wide association study Loci reveals new genes controlling hematopoiesis. PLoS Genet. 2014; 10(7):e1004450.

25. Bluteau O, Sebert M, Leblanc T, et al. A landscape of germ line mutations in cohort of inherited bone marrow failure patients. Blood. 2018;131(7):717-732 